\title{
Paroxysmal Sympathetic Hyperactivity: Ignoring the Presence of an Elephant in the Room
}

\author{
Parmod K. Bithal ${ }^{1} \quad$ Siddharth Chavali \\ ${ }^{1}$ Former Professor and Head, Department of Neuroanaesthesiology \\ and Critical Care, All India Institute of Medical Sciences, New Delhi, \\ India \\ ${ }^{2}$ Department of Neuroanaesthesia, Neurocritical Care and \\ Pain Medicine, Asian Institute of Gastroenterology Hospitals, \\ Hyderabad, Telangana, India
}

J Neuroanaesthesiol Crit Care 2021;8:161-162.

Paroxysmal sympathetic hyperactivity (PSH) is a syndrome of excessive and pathological adrenergic output to nociceptive or non-nociceptive (including environmental) stimuli. It is observed as a complication of various acute brain insults such as traumatic brain injury (TBI), stroke, anoxic brain injury, tumors, infections, autoimmune encephalitis, and acute hydrocephalus. It can manifest as a constellation of episodic, simultaneous symptoms such as tachycardia, hyperthermia, hypertension, tachypnea, and diaphoresis, often accompanied by dystonia and even motor posturing. ${ }^{1}$ Onset of these symptoms is usually fast, but resolution is slow, unless terminated by medication.

Since the first description of this syndrome by Penfield, ${ }^{2}$ many names have been ascribed to it which has created puzzlement in its diagnosis as well as understanding of its pathophysiology. Some of the names associated with this condition over the years are "autonomic storm," "sympathetic storm," "hypothalamic dysregulation syndrome," and "paroxysmal autonomic instability with dystonia." In 2014, the International Brain Injury Association proposed the term "paroxysmal sympathetic hyperactivity." 3 Its overall incidence is $18 \%$ among various cohorts of patients admitted in neurocritical care with an incidence of 33\% in severe TBI patients. ${ }^{4}$ According to Perkes et al, $80 \%$ of cases of PSH are observed after TBI, and the remaining $20 \%$ following other cerebral pathologies. ${ }^{5}$ The most consistent observation is that patients with PSH are frequently young and comatose. Pediatric patients appear more prone to develop PSH after anoxic-ischemic insults and with non-bacterial encephalitis. ${ }^{6}$ It is common that patients with PSH are erroneously suspected of having other diagnoses, and this may lead to unnecessary testing and sometimes inappropriate treatments, making an early and accurate diagnosis important.7 PSH may persist for weeks or months, and has been associated with worse clinical outcomes such as increased time of mechanical ventilation, infection, malnutrition, dehydration, tracheostomy, longer hospitalization longer intensive care unit (ICU) stays, contractures, and heterotopic ossification.

PSH remains an under-recognized condition that is difficult to diagnose. A high index of suspicion is key to early diagnosis. The first step in diagnosis is to exclude conditions with similar symptoms, such as infection, sedation withdrawal, seizures, and pulmonary embolism. Clinical diagnostic tools (PSH assessment measure) have been proposed to assist clinicians in the reliable identification of PSH. ${ }^{8}$ Such tools incorporate a clinical feature scale that categorizes the severity of sympathetic signs during episodes and a diagnostic tool that gauges the likelihood of diagnosis of PSH based on the presence of characteristic features. These two components are combined in a score that reflects the degree of confidence in diagnosis of PSH. The feasibility and reliability of these tools have been recently validated by van Eijck et al. ${ }^{9}$ There is evidence that they may reduce the chances of misdiagnosis and favorably impact hospital length of stay and costs of hospitalization. ${ }^{10}$

The pathophysiology of PSH is poorly understood and the dominant theory suggests the failure of the central autonomic network. Disruption of descending pathways releases sympathetic responses from their normal inhibitory modulation. The consequence is that sympathetic responses to internal or external stimuli become exaggerated. ${ }^{11}$ The interruption of descending inhibitory modulation might also produce maladaptive changes in the spinal cord leading to excitatory interneuronal activity. ${ }^{12}$ These changes could help explain how non-noxious stimuli are perceived as noxious by brain. ${ }^{12}$

While formal evidence on treatment is scant and lacks methodological quality, PSH is a disorder that can be treated. ${ }^{13}$ Can episodes be prevented with pharmacological intervention? There is at least one retrospective study that claims so. Tang et al asserted that dexmedetomidine infusion has

\author{
Address for correspondence \\ Siddharth Chavali, MD, DM, \\ Department of Neuroanaesthesia, \\ Neurocritical Care and Pain \\ Medicine, Asian Institute of \\ Gastroenterology Hospitals, \\ Hyderabad, Telangana 500082, India \\ (e-mail: sid.chavali@gmail.com). \\ DOI https://doi.org/ \\ $10.1055 / \mathrm{s}-0041-1740206$ \\ ISSN 2348-0548
}

\footnotetext{
C 2021. Indian Society of Neuroanaesthesiology and Critical Care. This is an open access article published by Thieme under the terms of the Creative Commons Attribution-NonDerivative-NonCommercial-License, permitting copying and reproduction so long as the original work is given appropriate credit. Contents may not be used for commercial purposes, or adapted, remixed, transformed or built upon. (https://creativecommons.org/licenses/by-nc-nd/4.0/). Thieme Medical and Scientific Publishers Pvt. Ltd. A-12, 2nd Floor, Sector 2, Noida-201301 UP, India
} 
preventive effect on PSH in severe TBI patients who have undergone surgery. ${ }^{14}$ Treatment revolves around meticulous general care and pharmacological therapy with abortive (morphine) and preventive medication (propranolol). Many other drugs have been used with varying success rates. Drug combinations are frequently used and chosen based on individual preference rather than objective evidence. Avoidance of triggering stimuli is critical in its management.

While there are numerous published studies from various countries to unravel the epidemiology, pathophysiology, and management of this serious but treatable condition, in India, neurocritical care specialists are still under-recognizing/under-reporting this complication. Failure to recognize the condition can adversely impact outcomes of these patients. Literature search in Indian context revealed a few case reports but only one small study on PSH incidence in 57 patients admitted in neurological ICU by Verma et al in 2015, in which the authors reported $10.53 \%$ incidence of PSH. ${ }^{15}$ In this issue of Journal of Neuroanesthesiology and Critical Care, Bhardwaj et al have published a retrospective study in a relatively large population of 257 patients with TBI, and have observed PSH in $30.8 \%$ of patients. ${ }^{16}$ This study focuses only on patients with TBI, which raises questions regarding the true incidence of PSH in other varieties of critical neurological diseases. Hence, to validate their reported incidence of $\mathrm{PSH}$, there is a crucial need for conducting prospective multicenter studies in the whole gamut of neurocritical care patients. This would expand the understanding of this condition among neurocritical care specialists with proper management and improved outcomes. A greater effort should also be devoted to discover pharmacological agents that can either prevent this dangerous syndrome or at least mitigate its detrimental effects. Simultaneously, another vital area of research could be to predict which patient is likely to develop this complication, through use of certain predictive clinical features and/or blood biomarkers unique to these vulnerable patients.

\section{Conflict of Interest}

None declared.

\section{References}

1 Pozzi M, Conti V, Locatelli F, et al. Paroxysmal sympathetic hyperactivity in pediatric rehabilitation: clinical factors and acute pharmacological management. J Head Trauma Rehabil 2015;30(5):357-363
2 Penfield W. Diencephalic autonomic epilepsy. Arch Neurol Psychiatry 1929;22:358-374

3 Baguley IJ, Perkes IE, Fernandez-Ortega JF, Rabinstein AA, Dolce G, Hendricks HT; Consensus Working Group. Paroxysmal sympathetic hyperactivity after acquired brain injury: consensus on conceptual definition, nomenclature, and diagnostic criteria. J Neurotrauma 2014;31(17):1515-1520

4 Rabinstein AA. Paroxysmal sympathetic hyperactivity in the neurological intensive care unit. Neurol Res 2007;29(7):680-682

5 Perkes I, Baguley IJ, Nott MT, Menon DK. A review of paroxysmal sympathetic hyperactivity after acquired brain injury. Ann Neurol 2010;68(2):126-135

6 Farias-Moeller R, Carpenter JL, Dean N, Wells EM. Paroxysmal sympathetic hyperactivity in critical ill children with encephalitis and meningoencephalitis. Neurocrit Care 2015;23(3):380-385

7 Rabinstein AA, Benarroch EE. Treatment of paroxysmal sympathetic hyperactivity. Curr Treat Options Neurol 2008;10(2):151-157

8 Baguley IJ, Perkes IE, Fernandez-Ortega J-F, et al. Consensus working group. Paroxysmal sympathetic hyperactivity after acquired brain injury: consensus on conceptual definition and diagnostic criteria. J Neurotrauma 2014;31:680-682

9 van Eijck MM, Sprengers MOP, Oldenbeuving AW, de Vries J, Schoonman GG, Roks G. The use of the PSH-AM in patients with diffuse axonal injury and autonomic dysregulation: a cohort study and review. J Crit Care 2019;49:110-117

10 Samuel S, Lee M, Brown RJ, Choi HA, Baguley IJ. Incidence of paroxysmal sympathetic hyperactivity following traumatic brain injury using assessment tools. Brain Inj 2018;32(9):1115-1121

11 Meyfroidt G, Baguley IJ, Menon DK. Paroxysmal sympathetic hyperactivity: the storm after acute brain injury. Lancet Neurol 2017;16(9):721-729

12 Baguley IJ. The excitatory:inhibitory ratio model (EIR model): an integrative explanation of acute autonomic overactivity syndromes. Med Hypotheses 2008;70(1):26-35

13 Scott RA, Rabinstein AA. Paroxysmal sympathetic hyperactivity. Semin Neurol 2020;40(5):485-491

14 Tang $\mathrm{Q}$ Wu X, Weng W, et al. The preventive effect of dexmedetomidine on paroxysmal sympathetic hyperactivity in severe traumatic brain injury patients who have undergone surgery: a retrospective study. PeerJ 2017;5:e298610.7717/peerj.2986

15 Verma R, Giri P, Rizvi I. Paroxysmal sympathetic hyperactivity in neurological critical care. Indian J Crit Care Med 2015;19(1):34-37

16 Bhardwaj A, Satpathy GC, Garg A, Chawla V, Jangra K. Incidence of paroxysmal sympathetic hyperactivity after traumatic brain injury in a tertiary care ICU: a retrospective study. J Neuroanesthesiol Crit Care 2021. Doi 10.1055/s-0040-1721553 